\title{
Concurrent Modified Constant Modulus Algorithm and Decision Directed Scheme with Barzilai-Borwein Method
}

Tongtong Xu ( $\sim$ ttxu@stu.xidian.edu.cn)

Xidian Univeristy https://orcid.org/0000-0002-8944-6134

Zheng Xiang

Xidian University

\section{Research}

Keywords: Blind equalization, Barzilai-Borwein method, Modified Constant Modulus Algorithm, DecisionDirected

Posted Date: February 14th, 2020

DOI: https://doi.org/10.21203/rs.2.23599/v1

License: (c) (i) This work is licensed under a Creative Commons Attribution 4.0 International License.

Read Full License

Version of Record: A version of this preprint was published at Frontiers in Neurorobotics on June 10th, 2021. See the published version at https://doi.org/10.3389/fnbot.2021.699221. 


\title{
Concurrent Modified Constant Modulus Algorithm and Decision Directed Scheme with Barzilai-Borwein Method
}

\author{
Tongtong $\mathrm{Xu}^{1 *}$, and Zheng Xiang ${ }^{1}$ \\ 1. Department of Telecommunication Engineering, Xidian University, Xi'an, 710071, China \\ Corresponding Author's Email: ttxu@stu.xidian.edu.cn
}

\begin{abstract}
In this work, concurrent modified constant modulus algorithm and decision-directed scheme with the Barzilai-Borwein method is proposed for blind equalization of wireless communications systems. The BarzilaiBorwein method, the two-step gradient method, is usually used to solve multidimensional unconstrained optimization problems. The proposed algorithm concurrently operates a modified constant modulus algorithm equalizer and a decision directed equalizer, and then adaptively adjusts the step size of the decision directed equalizer using Barzilai-Borwein method. Theoretical analysis is provided to illustrate that the proposed algorithm has a faster convergence speed, and better equalization performance than the original one. Simulation results support the new proposed technique.
\end{abstract}

Keywords: Blind equalization, Barzilai-Borwein method, Modified Constant Modulus Algorithm, Decision-Directed.

\section{Introduction}

It is well known that inter-symbol interference (ISI) is one of the major impairments to achieve a higher capacity or a data rate improvement in wireless communication systems. During the past years, various channel estimation algorithms and equalization methods [1]-[9] have been developed to overcome the effects of ISI and compensate the channel distortions. Blind equalization is a popular method for recovering transmitted symbols of superimposed noise without providing the desired response externally [3]. The constant modulus algorithm (CMA) [4] may be the most classical blind equalization algorithm because of its simplicity, stability, and efficiency. The approach, however, takes longer to converge and only could achieve a moderate level of mean square error (MSE) after convergence. Thus, it may not be sufficiently low to ensure adequate system performance, which is a serious weakness need to consider.

Researchers have putted forward a number of interesting solutions [5]-[9] to this problem. In [5] a variable step size (VSS) technique was developed and applied to CMA for 16 quadrature amplitude modulation (QAM) signals It improves the performance of the equalizer to some extent. However, it fails to take the higher-order QAM signals into account, which is another problem of this approach. On this basis, the modified constant modulus algorithm (MCMA) [5] was proposed, which could improve the performance of CMA by obtaining a low steady state MSE. It also could eliminate the need of joint carrier phase recovery. Another possible solution is switching to a decision directed (DD) mode, which has no need of a threshold level or estimating the state of convergence. It will be able to minimize the remaining CMA steady state MSE [7]. However, to make the transfer successful, the CMA steady state MSE must be low enough as pointed in [8]. In fact, MSE may not be achievable by the CMA as expected at such a low level.

In this paper, we will focus on the joint MCMA-DD equalization algorithm [9] and propose a concurrent MCMA and DD with the Barzilai-Borwein (BB) method [10] blind equalization algorithm for the wireless communication systems employing QAM signal. In [8], the authors have suggested to operate a DD equalizer concurrently with CMA equalizer, rather than switching to a DD scheme after the CMA has converged. The method is also applicable to MCMA. Concurrent MCMA and DD equalizers have significant improvements in equalization performance over MCMA. According to the blind equalizer (BE) output error, the proposed method could adjust the step size of the weight with updating formula by using BB method. It could furtherly improve the convergence speed, reduce the steady state MSE and remain the ISI.

\section{Blind Equalization}

\subsection{System Model}

For an adaptive blind channel equalization system, the received signal for the $k$ th symbol interval $x(k)$ can be expressed as

$$
x(k)=\sum_{i=0}^{L-1} h(i) s(k-i)+v(k)
$$

where $h(\mathrm{k})$ with order $\mathrm{L}$ is the impulse response of the channel. The transmitted sequence $s(k)$ takes the value from the QAM signal set. $v(k)$ is assumed to be additive white gaussian noise (AWGN) with zero mean and variance $\sigma^{2}$. The actual equalizer output can be written as 


$$
\hat{s(k)}=\boldsymbol{w}^{T}(k) \boldsymbol{x}(k)
$$

where $\boldsymbol{w}(k)=\left[w_{0}(k), w_{1}(k), \cdots, w_{N-1}(k)\right]^{T}$ is the weight vector of the $\mathrm{BE}$ with order $\mathrm{N}$ and $\boldsymbol{x}(k)=[x(k), x(k-1), \cdots, x(k-N+1)]^{T}$ is the received sample vector.

\subsection{Concurrent MCMA and DD} the form

The MCMA changes the cost function of CMA [4] from real field to complex field, and its cost function has

$$
\psi_{M C M A}(k)=\psi_{M C M A, R}(k)+\psi_{M C M A, I}(k)
$$

where $\psi_{\mathrm{MCMA}, \mathrm{R}}(k)$ and $\psi_{\mathrm{MCMA}, \mathrm{I}}(k)$ are, respectively, the cost function for real and imaginary parts.

The MCMA equalizer output $\hat{s(k)}$ can be expressed as

$$
\hat{s(k)}=\boldsymbol{w}_{M C M A}^{T}(k) \boldsymbol{x}(k)
$$

The equalizer weights vector $\boldsymbol{w}_{M C M A}(k)$ is updated according to the rule

where $\mu_{M C M A}$ is the step size.

$$
\begin{aligned}
\boldsymbol{w}_{M C M A}(k+1) & =\boldsymbol{w}_{M C M A}(k)-\mu_{M C M A} \nabla \psi_{M C M A}(k) \\
& =\boldsymbol{w}_{M C M A}(k)-\mu_{M C M A} e_{M C M A}(k) \boldsymbol{x}^{*}(k)
\end{aligned}
$$

The error signal $e_{M C M A}(k)=e_{M C M A, R}(k)+j e_{M C M A, I}(k)$ is given by

$$
\begin{aligned}
& e_{M C M A, R}(k)=\hat{s_{R}} \hat{(k)}\left(\left|\hat{s_{R}} \hat{(k)}\right|^{2}-R_{R}\right) \\
& e_{M C M A, I}(k)=\hat{s_{I}} \hat{(k)}\left(\left|\hat{s_{I}(k)}\right|^{2}-R_{I}\right)
\end{aligned}
$$

where $R$ is a normal number.

It is not difficult to see that the MCMA comprehensively considers the amplitude and phase equalization, which can correct the phase deflection during transmission to a certain extent and achieve a better equalization effect.

The concurrent MCMA and DD algorithm consists of a MCMA equalizer and a DD equalizer operating concurrently. Explicitly, let $\boldsymbol{w}=\boldsymbol{w}_{M C M A}+\boldsymbol{w}_{D D}$, where $\boldsymbol{w}_{D D}$ is the weight vector of the DD equalizer.

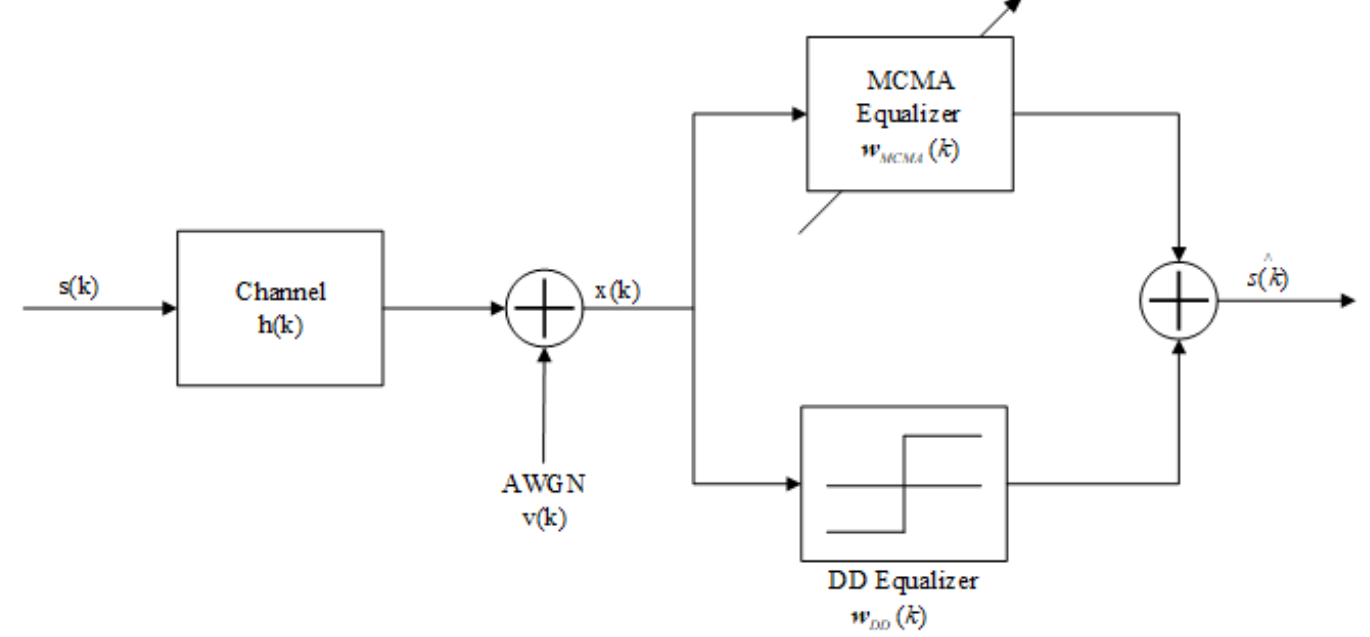

Fig. 1 Baseband model of the concurrent MCMA and DD blind channel equalization system.

Fig. 1 shows the baseband model of the concurrent MCMA and DD blind channel equalization system. The cost function of the DD equalizer is can be described as

$$
\psi_{D D}(k)=E\left[\mid\left[|[s(\hat{k})]-s(\hat{k})|^{2}\right]\right.
$$

where $\llbracket[\hat{s(k)}]$ denotes the quantized MCMA equalizer output defined by 


$$
\mathbb{I}[s(\hat{k})]=\arg \min _{s(k) \in S}|s(k)-s(k)|^{2}
$$

The DD adaptation is performed immediately after MCMA adaptation. The equalizer output can be expressed as

$$
s(k)=\boldsymbol{w}_{M C M A}^{T}(k+1) \boldsymbol{x}(k)+\boldsymbol{w}_{D D}^{T}(k) \boldsymbol{x}(k)
$$

The DD equalizer updated coefficient is written as

$$
\begin{aligned}
\boldsymbol{w}_{D D}(k+1)= & \boldsymbol{w}_{D D}(k)-\mu_{D D} \nabla \psi_{D D}(k) \\
& =\boldsymbol{w}_{D D}(k)-2 \mu_{D D} e_{D D}(k) \boldsymbol{x}^{*}(k)
\end{aligned}
$$

where $\mu_{D D}$ is the step size of the DD equalizer and $e_{D D}(k)=s(k)-\llbracket[(s(k))]$ is the value of the estimated error.

It could be seen that the updated $\boldsymbol{w}_{D D}$ occurs after the CMA equalization and before the equalizer hard decision. Compared to pure MCMA, Concurrent MCMA and DD adaptation has a faster convergence speed and a lower steady state MSE. The $\mu_{D D}$ for the DD equalizer can often be chosen much larger than $\mu_{M C M A}$ for the MCMA.

\section{The Proposed Concurrent MCMA and DD with BB Method}

\subsection{The Barzilai-Borwein Method}

For multidimensional unconstrained optimization problems, i.e. $\min _{x \in \mathfrak{R}_{n}} f(x)$, the gradient method is the simplest solution, which uses the negative gradient direction as the search direction. The iteration formula for this method is

$$
x_{k+1}=x_{k}-\lambda_{k} g_{k}
$$

where $\lambda_{k}$ is the iteration step and $g_{k}=\nabla f\left(x_{k}\right)$ is the search direction.

The BB method, also known as the two-step gradient method, was initially proposed by Barzilai and Borwein in [10] It was applied to solve various unconstrained optimization problems [11]-[12] lately. The method has a faster convergence rate than the gradient method.

The basic idea of the BB method is to use the information of the current point and the previous point to determine the step factor and convert (12) to

$$
x_{k+1}=x_{k}-D_{k} g_{k}
$$

where $D_{k}=\lambda_{k} I, I$ is the unit matrix. In order to make $D_{k}$ has "quasi-Newton" properties, calculate $\lambda_{k}$ to satisfy the formula

or

$$
\min \left\|d_{k-1}-D_{k} \tilde{g}_{k-1}\right\|_{2}
$$

$$
\min \left\|D_{k}^{-1} d_{k-1}-\tilde{g}_{k-1}\right\|_{2}
$$

where $d_{k-1}=x_{k}-x_{k-1}$ and $\tilde{g}_{k-1}=g_{k}-g_{k-1}$.

From (13) and (14), the formulas

$$
\lambda_{k}=d_{k-1}^{T} \tilde{g}_{k-1} /\left\|\tilde{g}_{k-1}\right\|_{2}^{2}
$$

and

$$
\lambda_{k}=\left\|d_{k-1}\right\|_{2}^{2} / d_{k-1}^{T} \tilde{g}_{k-1}
$$

can be obtained.

Barzilai and Borwein had proved that the algorithm, whose step size is determined by (16) and (17), is linearly convergent in R domain, and the convergence order is $\sqrt{2}$. The result is true for almost initial values.

\subsection{The Concurrent MCMA and DD with BB Method}

We considered applying formula (17) to update the value of $\mu_{D D}$ in concurrent MCMA and DD algorithm, which could improve the convergence speed. Thus $\mu_{D D}(k)$ could be written as

$$
\mu_{D D}(k)=\|d(k)\|_{2}^{2} / d^{T}(k) \tilde{g}(k)
$$

where

$$
d(k)=x(k+1)-x(k)
$$

and

$$
\begin{aligned}
\tilde{g}(k) & =\nabla \psi_{D D}(k+1)-\nabla \psi_{D D}(k) \\
& =2 e_{D D}(k+1) \boldsymbol{x}^{*}(k+1)-2 e_{D D}(k) \boldsymbol{x}^{*}(k)
\end{aligned}
$$


The formula (11) can be rewritten as

$$
\begin{aligned}
\boldsymbol{w}_{D D}(k+1) & =\boldsymbol{w}_{D D}(k)-\mu_{D D}(k) \nabla \psi_{D D}(k) \\
& =\boldsymbol{w}_{D D}(k)-2 \mu_{D D}(k) e_{D D}(k) \boldsymbol{x}^{*}(k)
\end{aligned}
$$

The entire blind equalization process can be summarized in the form of Algorithm 1.

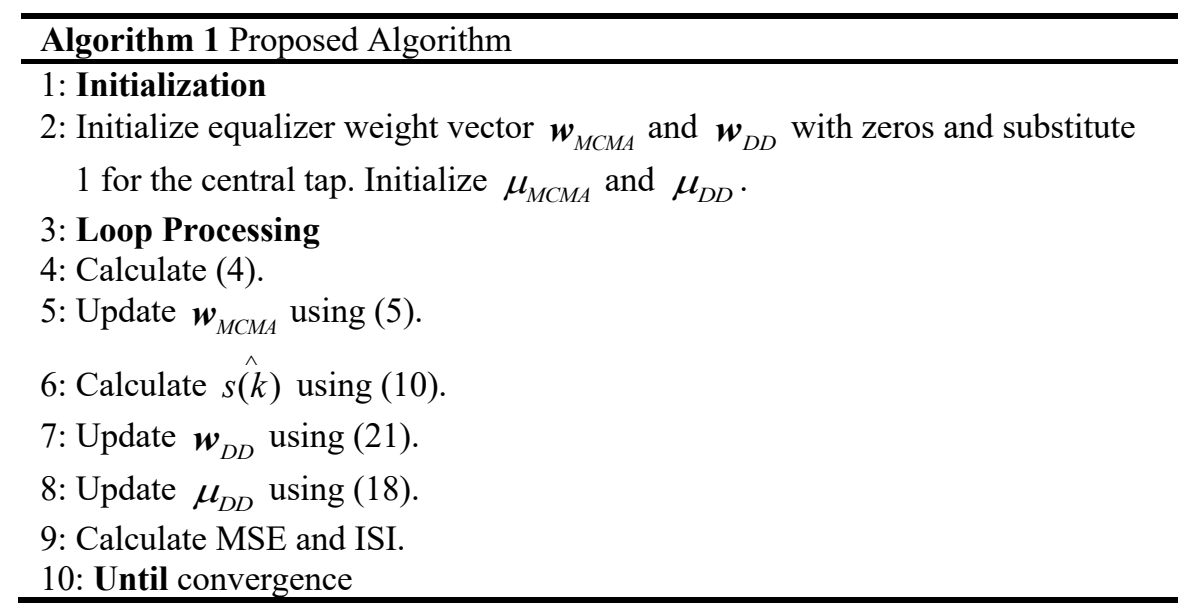

From what has been discussed above, it is obvious that the value of $\mu_{D D}$ is mainly related to $\tilde{g}(k)$. MCMA has performed better in the initial stage of the equalization iteration and plays a leading role at this stage. The difference of the estimation error decreases gradually but the $\mu_{D D}$ gradually increases with the progress of the equalization iteration process, which makes DD taking the dominant position. Thus, the convergence rate has been accelerated and the steady-state MSE has been reduced furtherly.

\section{Simulation Results and Performance Analysis}

The performance of the proposed concurrent MCMA and DD with BB algorithm was demonstrated by means of simulations. Two criteria were used to estimate the performance of the proposed algorithm. The first one was MSE. The MSE was defined as

$$
M S E=\frac{1}{N} \sum_{k=1}^{N}|\llbracket[s(\hat{k})]-s \hat{(k)}|^{2}
$$

The second one was ISI defined by

$$
I S I=\frac{\sum_{k=1}^{N}\left(\left|h(k) * \boldsymbol{w}^{*}(k)\right|\right)-\left|h(k) * \boldsymbol{w}^{*}(k)\right|_{\max }}{\left|h(k)^{*} \boldsymbol{w}^{*}(k)\right|_{\max }}
$$

The performance simulations of MCMA, concurrent MCMA-DD and concurrent MCMA-DD with BB were carried out in a 30-dB SNR environment with 64-QAM. The channel impulse response was set to be $\mathbf{h}=$ $[0.005,0.009,-0.0024,0.854,-0.218,0.049,-0.016]^{\mathrm{T}}$, then the channel was normalized.

The step size of the MCMA had to be set to $\mu_{M C M A}=2 \times 10^{-6}$. The two step sizes of the concurrent MCMADD equalizer were set to $\mu_{M C M A}=2 \times 10^{-6}$ and $\mu_{D D}=10^{-4}$. For the concurrent MCMA-DD with BB equalizer, the two step sizes were set to $\mu_{M C M A}=2 \times 10^{-6}$ and the initial $\mu_{D D}=10^{-3}$. 


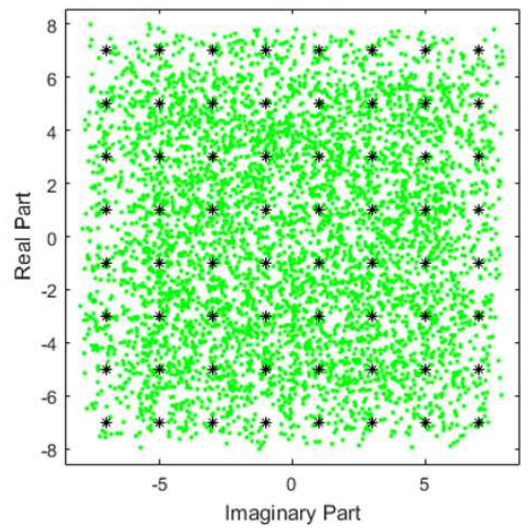

(a)

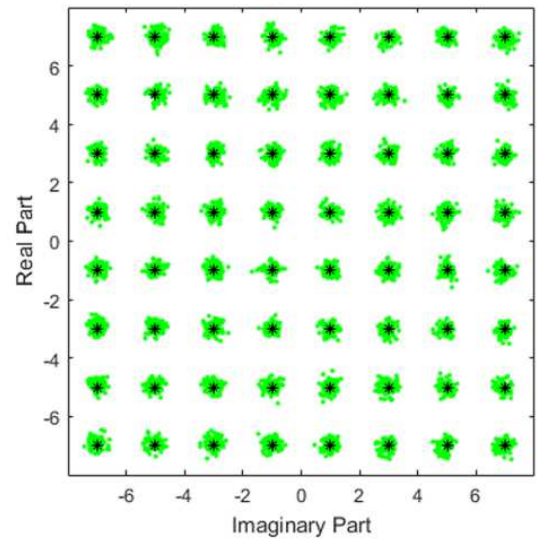

(b)

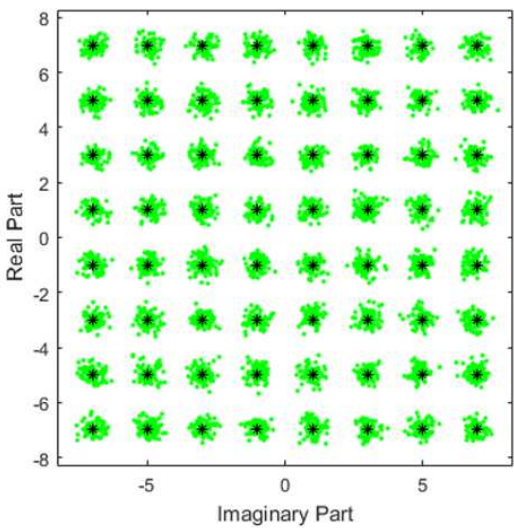

(b)

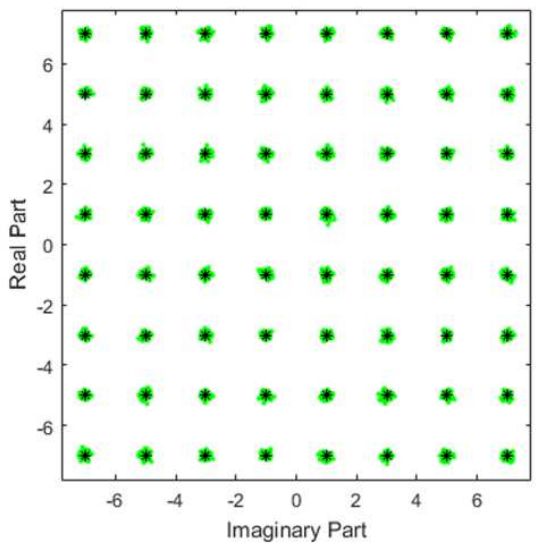

(d)

Fig. 2 (a) The equalizer input signal diagram and the output constellation diagrams of (b) MCMA, (c) Concurrent MCMA-DD and (d) Concurrent MCMA-DD with BB.

Fig. 2 shows the equalizer input signal diagram and the output constellation diagrams of the three algorithms. It could be intuitively seen that the proposed concurrent MCMA-DD with BB algorithm compensates the channel distortion more effectively comparing with other algorithms.

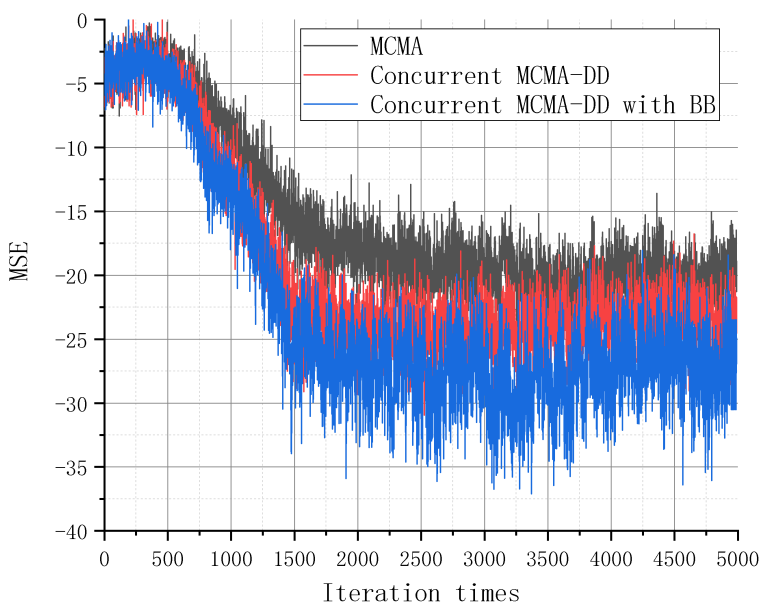

Fig. 3 The MSE performance of the three equalizers. 


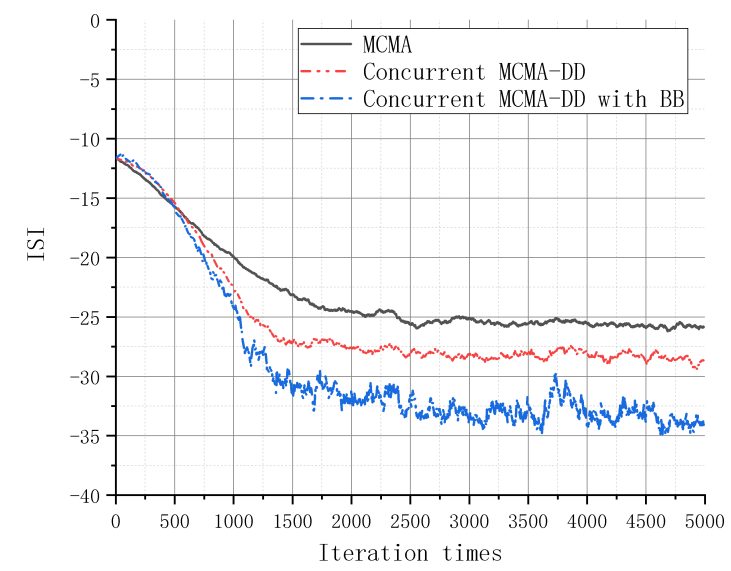

Fig. 4 The ISI performance of the three equalizers.

The learning curves for the three equalizers are shown in Fig. 3 and Fig. 4 based on the estimated MSE and ISI measurements. The results indicate that the convergence speed and the steady-state equalization performance of the concurrent MCMA-DD with BB algorithm is better than the MCMA and the concurrent MCMA-DD algorithm. Specifically, compared to the concurrent MCMA-DD algorithm, the convergence rate of the proposed algorithm is nearly 100 symbols faster, and steady-state error is reduced by $5 \mathrm{~dB}$.

\section{Conclusion}

In this paper, a concurrent MCMA and DD with $\mathrm{BB}$ algorithm has been proposed. Compared with MCMA and concurrent MCMA-DD, the proposed algorithm has a robust equalization performance and fast convergence speed. The proposed algorithm could be applied to some more complex wireless channel environments, which are of great significance for improving the adaptability of wireless channels.

\section{Abbreviation}

ISI: inter-symbol interference

CMA: constant modulus algorithm

QAM: quadrature amplitude modulation

MCMA: modified constant modulus algorithm

DD: decision directed

BB: Barzilai-Borwein

BE: blind equalizer

AWGN: gaussian noise

\section{Declarations}

\section{Availability of data and materials}

Not applicable.

\section{Competing interests}

The authors declare that they have no competing interests.

\section{Funding}

The work of the authors was supported by the natural science basic research plan in Shaanxi Province of China (Program No. 2019JQ-383).

\section{Authors' contributions}

Tongtong $\mathrm{Xu}$ is the main writer of this paper. He proposed the main idea, analyzed the feasibility and of the algorithm, completed the simulation. Zheng Xiang gave some important suggestions for algorithm idea. All authors read and approved the final manuscript. 


\section{References}

[1] S Haykin, The least-mean-square (LMS) algorithm, Adaptive filter theory, 5th edn, New Jersey, USA: Pearson Education. 248-308(2015).

[2] SM Shah, R Samar, NM Khan, and MAZ Raja, Design of fractional-order variants of complex LMS and NLMS algorithms for adaptive channel equalization, Nonlinear Dynamics. 88(2), 839-858(2017).

[3] J Yang, JJ Werner, and GA Dumont, The multimodulus blind equalization and its generalized algorithms, IEEE Journal on selected areas in communications. 20(5), 997-1015(2002).

[4] R Johnson, P Schniter, TJ Endres, and JD Behm, "Blind equalization using the constant modulus criterion: A review," Proceedings of the IEEE, vol. 86, no. 10, pp. 1927-1950(1998).

[5] Z Liyi, C Lei, and S Yunshan, Variable step-size CMA blind equalization based on non-linear function of error signal, 2009 WRI International Conference on Communications and Mobile Computing, Yunnan, China. 396-399(2009).

[6] KN Oh, and YO Chin, Modified constant modulus algorithm: blind equalization and carrier phase recovery algorithm, IEEE International Conference on Communications, USA. 498-502(1995).

[7] O Macchi, and E Eweda, Convergence analysis of self-adaptive equalizers, IEEE Transactions on Information Theory. 30(2), 161-176(1984).

[8] S Chen, and ES Chng, Concurrent constant modulus algorithm and soft decision directed scheme for fractionally-spaced blind equalization, 2004 IEEE International Conference on Communications, Paris, France. 2342-2346(2004).

[9] D Ashmawy, K Banovic, and E Abdel-Raheem, et al, Joint MCMA and DD blind equalization algorithm with variable-step size, 2009 IEEE International Conference on Electro/Information Technology, Canada. 174-177(2009).

[10] J Barzilai, and JM Borwein, Two-point step size gradient methods, IMA journal of numerical analysis. 8(1), 141-148(1988).

[11] C Tan, S Ma, YH Dai, and Y Qian, Barzilai-Borwein step size for stochastic gradient descent, Advances in Neural Information Processing Systems, Vancouver, B.C., Canada. 685-693(2016).

[12] YH Dai, WW Hager, K Schittkowski, and H Zhang, The cyclic Barzilai--Borwein method for unconstrained optimization, IMA Journal of Numerical Analysis. 26(3), 604-627(2006). 
Figures

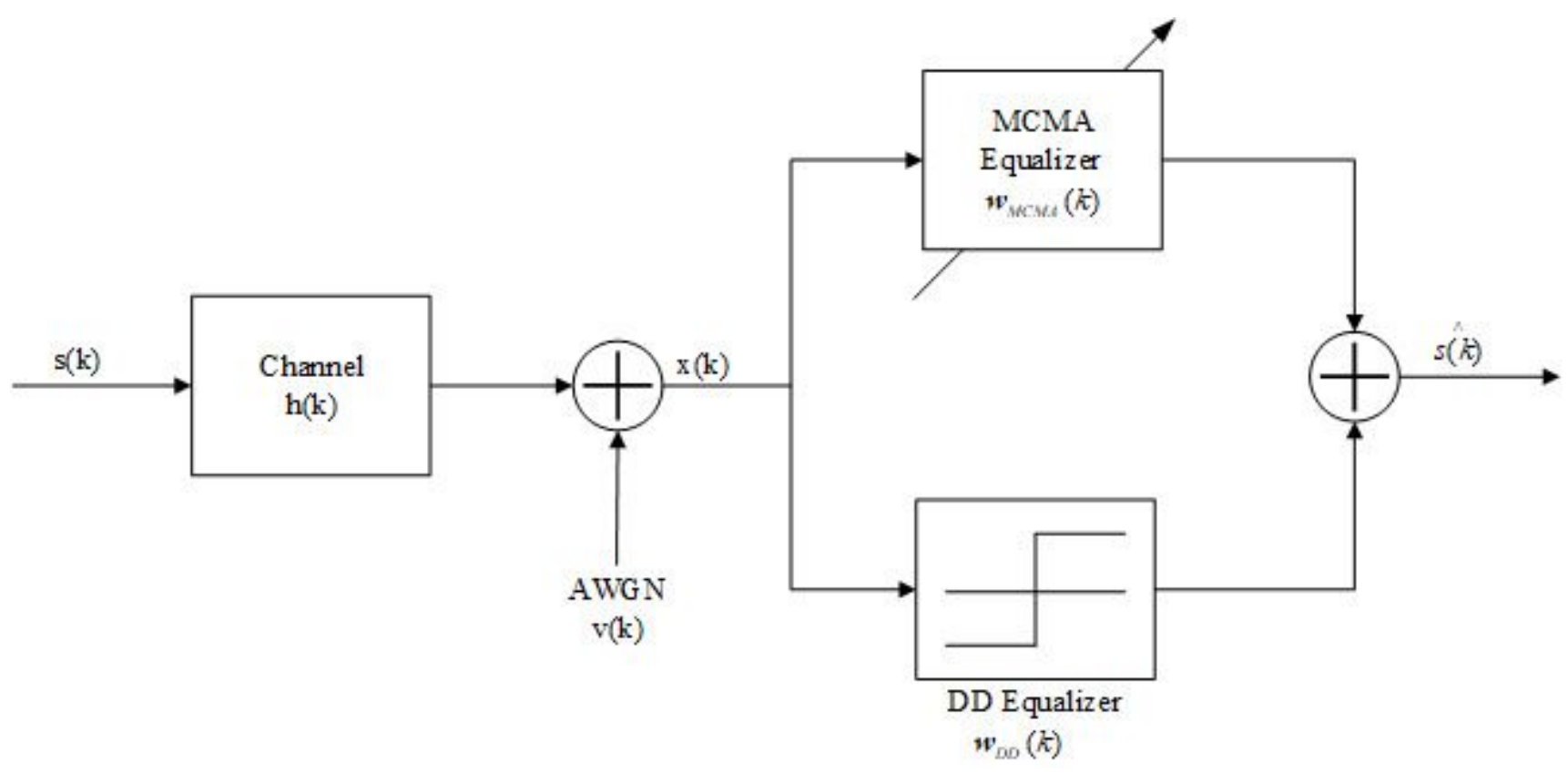

Figure 1

Baseband model of the concurrent MCMA and DD blind channel equalization system 
A

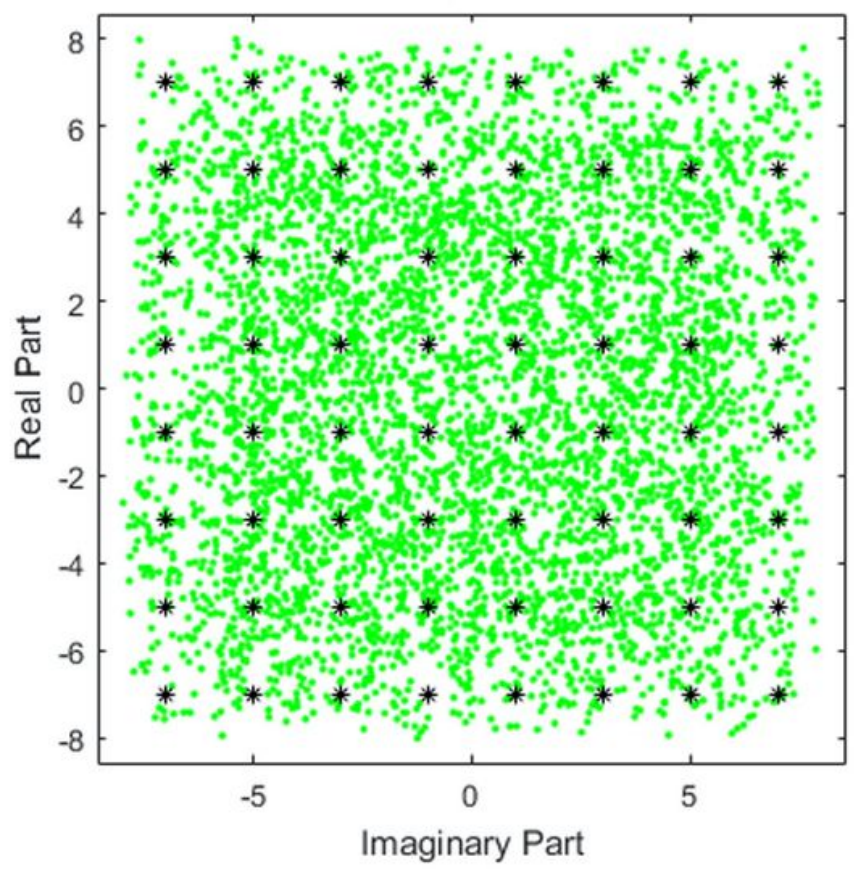

c

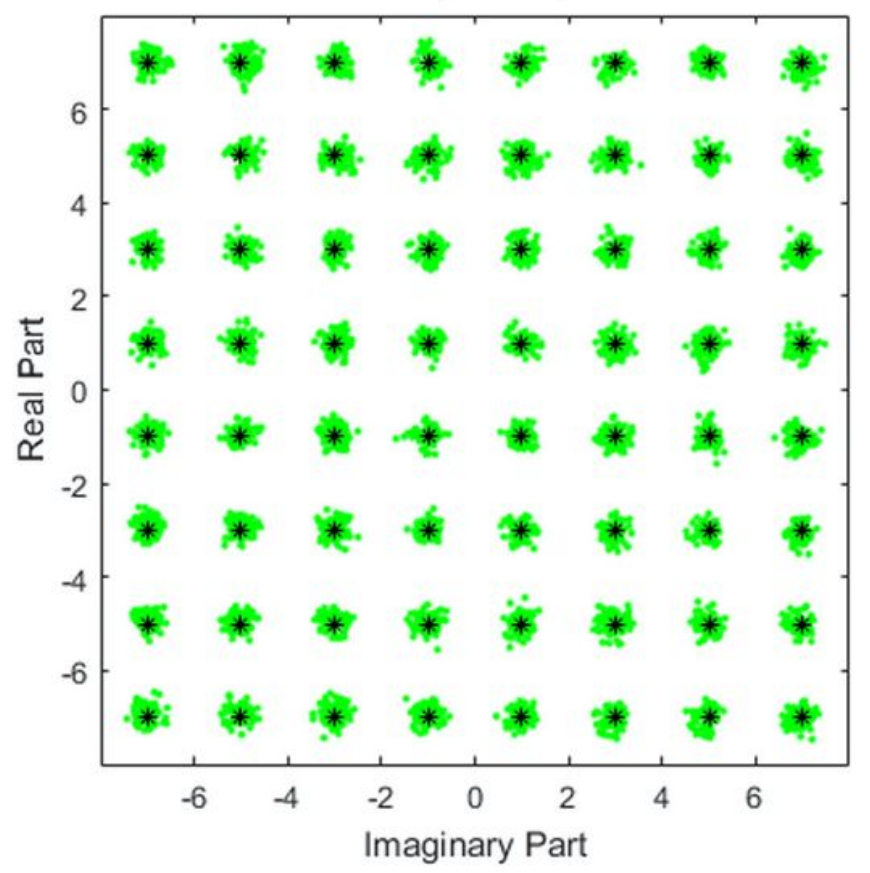

B

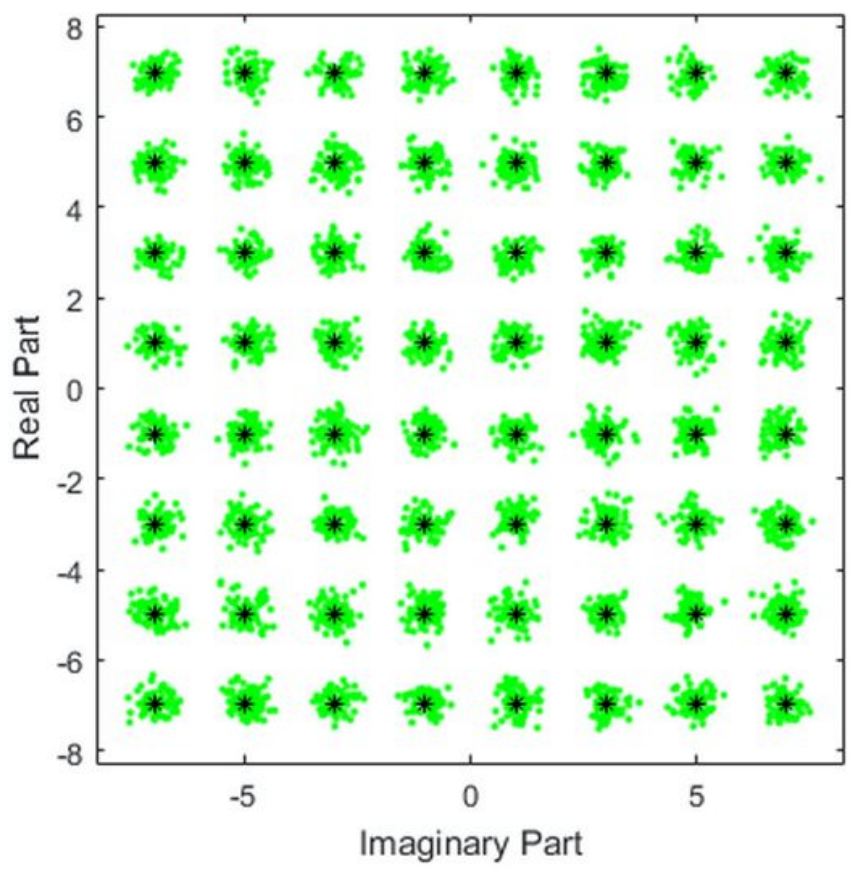

D

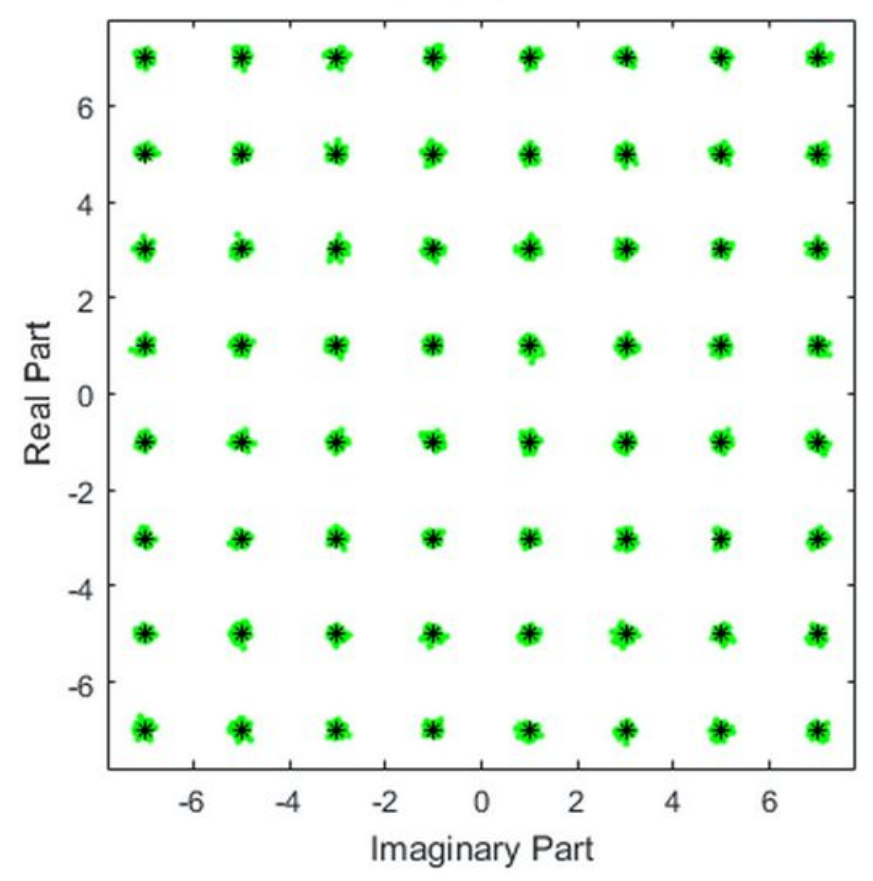

Figure 2

(a) The equalizer input signal diagram and the output constellation diagrams of (b) MCMA, (c) Concurrent MCMA-DD and (d) Concurrent MCMA-DD with BB. 


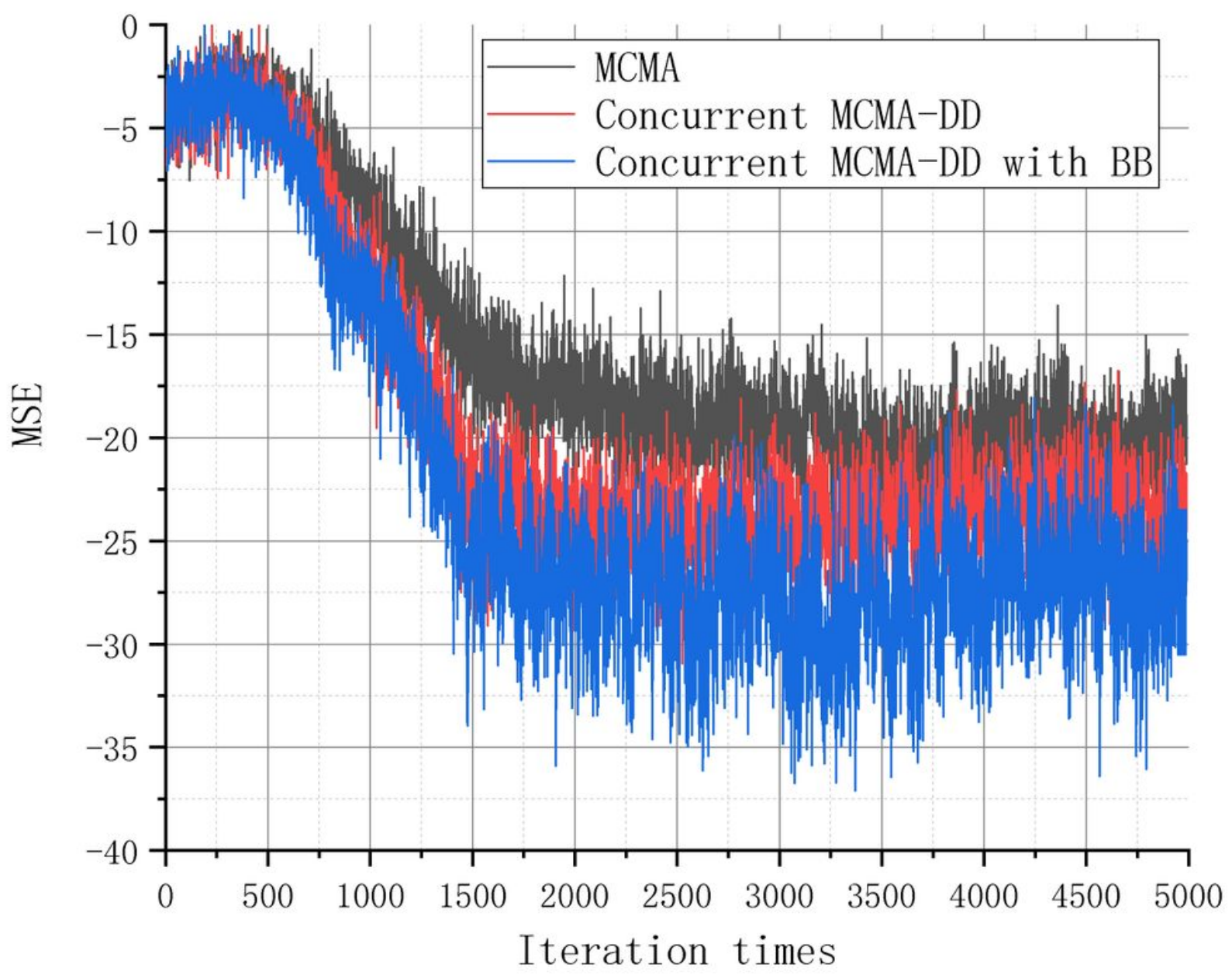

Figure 3

The MSE performance of the three equalizers. 


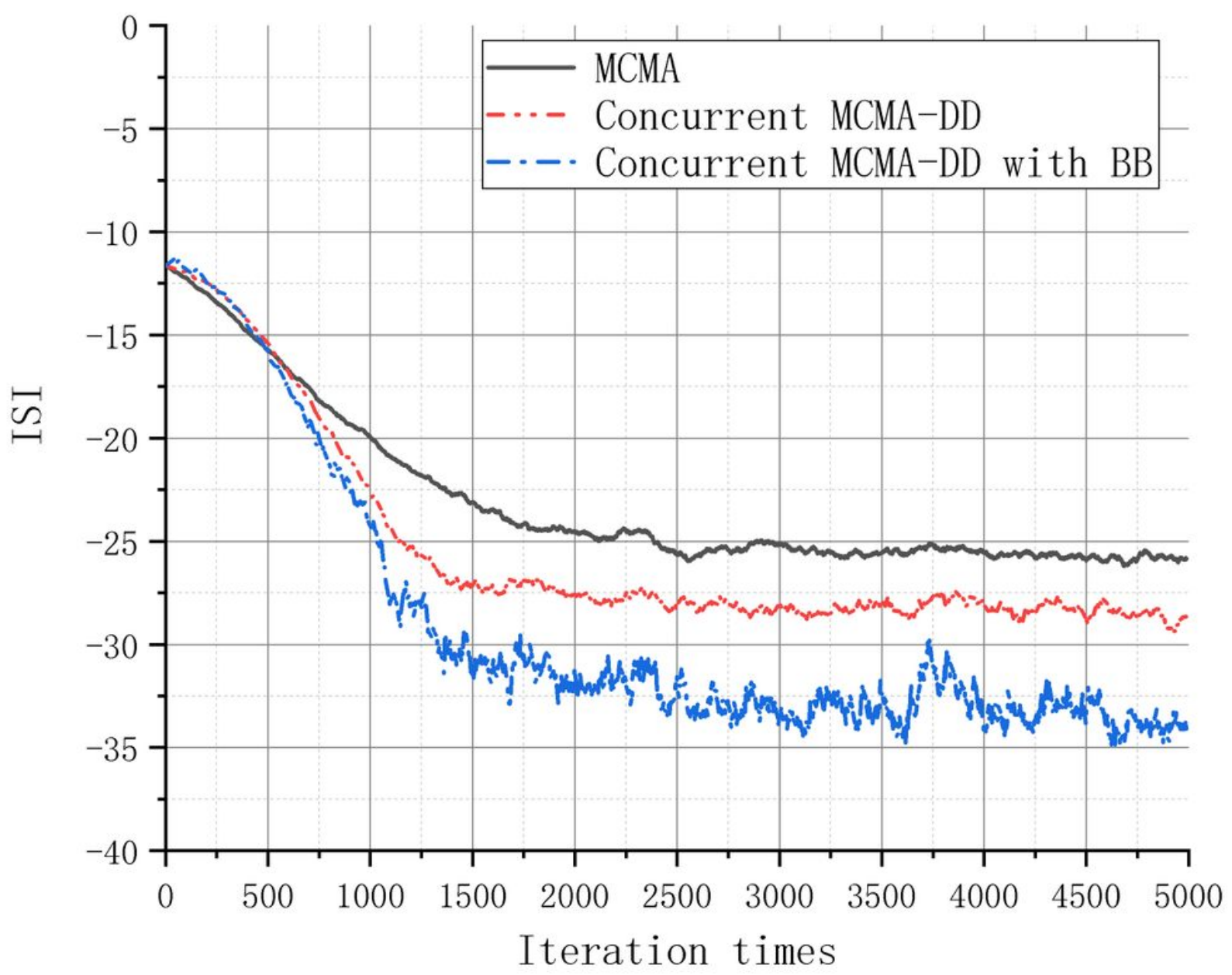

Figure 4

The ISI performance of the three equalizers. 